\title{
OBITUARY
}

\section{Jean Kérisel}

Jean Kérisel, past president of the ISSMFE (1973-77), died in Paris on 22 January 2005. He was recently very happy to see his last book, Of Stones and Man: From the Pharaohs to the Present Day, published on 26 December 2004. The English version of this book will be available soon.

A man who always remained young in spirit and who deeply loved life, Jean Kérisel kept an excellent balance between family and professional life. He was open minded, always ready to listen, and exhibited great strength of character.

Jean Kérisel was born in St Brieuc on the northern coast of Brittany. A student of the Ecole Polytechnique, he graduated in 1933 at Ecole Nationale des Ponts et Chaussées as a civil servant in the Corps des Ponts et Chaussées. He was awarded a $\mathrm{PhD}$ at the Sorbonne in 1935 on 'Friction of cohesionless soils and its application to the design of foundations'.

During the Second World War he was an officer of the French Army Corps of Engineers, and was awarded the French Croix de Guerre. After the war he resumed his duties with the Civil Corps, having important responsibilities in the Ministry of Reconstruction.

His life was much affected by his encounter with the famous engineer and scientist Professor Albert Caquot. He married Professor Caquot's daughter in 1931, and published with Caquot the first Manual of soil mechanics in French in 1949. He also published in 1948 the Tables for the calculation of passive pressure, active pressure and bearing capacity of foundations, which have been translated into many languages and frequently re-published. In 1952, he succeeded Professor Caquot as Professor of Soil Mechanics and Foundation Engineering at the Ecole Nationale des Ponts et Chaussées, and at the same time he founded his own geotechnical engineering company SIMECSOL.

Jean Kérisel was a consultant on many large projects all over the world, and he was very active in research. On in situ testing he initiated a scientific contest on cone penetration in France in 1948, and later supported the work of Louis Menard on the pressuremeter. In 1975 he gave the 15th Rankine Lecture on the theme 'Old structures in relation to soil conditions'. He was Chairman of the French Society of Civil Engineers 1968-69, Chairman of the French Geotechnical Society (CFMS) 1969-73, and most notably Chairman of the International Society of Soil Mechanics and Foundation Engineering 1973-77.

From 1979 onwards he devoted the time he did not keep for his large family to research and writing on the builders of Ancient Egypt. He published various books, two of them translated in English: Down to earth-Foundations past and present: the invisible art of the builder (1987), and The Nile and its masters: Past, present, future. Source of hope and anger (2001). He also wrote Albert Caquot, 1881-1976, Savant, soldat, bâtisseur (2001) showing his admiration for his father-in-law.

Jean Kérisel leaves three children, 12 grandchildren and 23 great-grandchildren. His wife pre-deceased him in 1998.

M. B. de la Motte and M. Gambin
Jean Kérisel, ancien Président de la SIMSTF (1973-1977) est décédé à Paris le 22 janvier 2005. Une de ses dernières grandes joies a été la publication récente de son dernier ouvrage Pierre et Hommes, des Pharaons à nos Jours, le 26 décembre 2004, dont la version anglaise sera bientôt disponible.

Jean Kérisel était un homme qui est toujours resté jeune et qui aimait profondément la vie. Il a su conduire avec une égale perfection et un parfait équilibre sa vie personnelle, familiale et professionnelle. D'une grande intelligence, d'une large ouverture d'esprit, d'une grande qualité de coeur à l'écoute de tous, il était d'un courage et d'une volonté qui ne se sont jamais démentis.

Jean Kérisel est né à St Brieuc sur la côte nord de la Bretagne, au bord de la Manche. Après des études à l'Ecole Polytechnique, il intègre le corps des Ponts et Chaussées et obtient le diplôme de l'Ecole Nationale des Ponts et Chaussées en 1933. Il obtient un doctorat à la Sorbonne sur 'L'étude des milieux pulvérulents et son application à l'étude des fondations'.

Pendant la seconde guerre mondiale, il fut officier du Génie et a été décoré de la Croix de Guerre. Il a poursuivi sa carrière après la guerre en assumant des postes de responsabilité au Ministère de la Reconstruction.

Sa vie a été marquée par sa rencontre avec le grand savant et ingénieur Albert Caquot, dont il épouse la fille en 1931. Il publiera avec Caquot le fameux Traité de mécanique des sols en 1949, ainsi que les Tables de poussée, de butée et de force portante des fondations (1948) qui ont été traduites en de nombreuses langues et souvent republiées. Il succède en 1952 à Caquot comme Professeur de Mécanique des sols à l'Ecole Nationale des Ponts et Chaussées et fonde à la même époque le bureau d'ingénieurs conseils SIMECSOL.

Jean Kérisel fut impliqué comme expert dans de nombreux projets dans le monde entier et il eut également une importante activité de recherche. Il lance un concours sur les méthodes de mesure de la résistance des sols à la pénétration et encourage les développements de Louis Ménard sur le pressiomètre. Il donne en 1975 la 15ème Rankine Lecture sur le thème 'Old structure in relation to soil conditions'. Il fut président de la Société des Ingénieurs Civils de France 1968-1969, du Comité Français de mécanique des Sols 1969-1973 et de la Société Internationale de Mécanique des Sols et des Travaux de Fondations 1973-1977.

Depuis 1979 , il consacre le temps qu'il ne voue pas à sa grande famille à des travaux sur la construction dans l'Ancienne Egypte et publie divers ouvrages dont plusieurs ont été traduits en Anglais: Down to earth - Foundations past and present: the invisible art of the builder (1987), La pyramide à travers les ages, Mythes et Religions (1991), Génie et démesure d'un grand pharaon : Khéops (1996), Le Nil : l'espoir et la colère (1999) et Pierre et hommes, des Pharaons à nos jours (2004). Pour exprimer sa grande admiration envers son beau-père, il écrit également un livre intitulé Albert Caquot, 1881-1976, Savant, soldat, bâtisseur (2001).

Jean Kérisel laisse trois enfants, douze petits-enfants et vingt-trois arrière petits enfants, auxquel nous exprimons nos plus vives condoléances. Sa femme était décédée en 1998.

M. B. de la Motte et M. Gambin 
JEAN KERISEL'S PUBLICATIONS

Papers published as a civil servant (Bibliothèque Nationale, Paris)

1946 Le plan de reconstruction et ses exigences économiques et financières.

1948 Point actuel de la reconstruction, ses perspectives d'aveni.

1946 Le problème de 1'habitation du point de vue industriel.

1950 Problèmes généraux de la construction et de la reconstruction en France et en Grande Bretagne.

Papers on underground urbanism

1968 La rénovation de Paris. La Revue Economique Française 1.

1968 L'urbanisme souterrain. Revue de la Société Royale Belge des Ingénieurs et des Industriels 3, mars 1969.

PhD thesis

1935 Contribution à 1'étude du frottement des milieux pulvérulents et application à l'étude des fondations. Thèse de Doctorat en Sorbonne, 3 juillet 1935, Paris: Gauthier-Villars.

1935 L’hystérésis dans les phénomènes mécaniques. Thèse de Doctorat en Sorbonne, 3 juillet 1935, Paris: Gauthier-Villars.

\section{Technical papers}

1937 L'hystérésis dans les milieux pulvérulents. Annales des Ponts et Chaussées 23, novembre 1938.

1939 La force portante des pieux. Annales des Ponts et Chaussées.

1948 (with A. Caquot) Théorie générale de la force portante des pieux. Revue Travaux, juin.

1952 (with A Caquot) Sur le terme de surface dans le calcul des fondations en milieu pulvérulent. Annales de I'Institut Technique du Bâtiment et des Travaux Publics, mars-avril 1953.

1952 (with A Caquot) Courbes de glissement de sol sous la pointe des pieux. Annales de I'Institut Technique du Bâtiment et des Travaux Publics, mars-avril 1953.

1953 Déformations et contraintes au voisinage des pieux. Association Belge pour 1'étude, l'essai et 1'emploi des matériaux, Bruxelles.

1956 Historique de la mécanique des sols en France jusqu'au 20ème siècle. Géotechnique 6, No. 4, $151-166$.

1957 Soil mechanics and foundation engineering. Comptes-Rendus du 4ème Congrès International de Mécanique des Sols et des Travaux de Fondations, Londres.

1957 Mesures in situ pour déterminer la portance d'une fondation: interprétation des resultats du pénétromètre. Cahiers du Centre Scientifique et Technique du Bâtiment 30, No. 254, 1-22.

1957 Poussée des terres sur les ouvrages et tunnels. Comptes-Rendus du 4ème Congrès International de Mécanique des Sols et des Travaux de Fondations, Londres.

1958 Fondations profondes en terrains médiocres. Annales de l'Institut Technique du Bâtiment et des Travaux Publics, fevrier.

1958 Puits d'essai du pont rail-route d'Abidjan. Revue Travaux, avril.

1958 La mécanique des sols: recherches et investigations récentes. Revue Travaux, septembre.
1961 Fondations profondes en milieu sableux. ComptesRendus du 5ème Congrès International de Mécanique des Sols et des Travaux de Fondations, Paris 2, 73.

1962 Résumé de quelques experiences de fondations profondes dans des sols pulvérulents. Bulletin no. 7 de la Sociedad Venezolana de Mecanica del Suelo e Ingenieria de Fundaciones, avril-mai-juin 1962.

1962 (with M. Adam) Fondations profondes. Annales de l'Institut Technique du Bâtiment et des Travaux Publics, novembre 1962, no. 179.

1962 Barrages en terre, leur conception et leur calcul. Coopération Technique, A.S.T.E.F., avril-juin 1962.

1963 Le pont en béton précontraint de $8.678 \mathrm{~m}$ de longueur sur le Lac Maracaïbo (Venezuela). Revue la Technique des Travaux, septembre-octobre.

1963 Stabilisation des sols par effet progressif de dalle dans l'utilisation des laitiers.

1963 Necessité de rapporter les tassements au rayon moyen de la surface chargée et les pressions appliquées aux pressions limites. Congrès européen de mécanique des sols et travaux de fondations, Wiesbaden.

1964 Evolution de la mécanique des sols, extrait du volume d'hommage au Professeur F. Campus.

1964 Deep foundations: basic experimental facts. Deep foundations conference, Mexico.

1965 (with R. L'Herminier et Y. Tcheng) Resistance de pointe en milieux pulvérulents de serrages divers. Comptes-Rendus du Gème Congrès International de Mécanique des Sols et des Travaux de Fondations, Montréal.

1965 Vertical and horizontal bearing capacity of deep foundations in clay. Conférence donnée à Duke University, USA.

1965 (with A. Caquot) Classification des propriétés des argiles saturées en fonction de l'indice des vides. Comptes-Rendus du bème Congrès International de Mécanique des Sols et des Travaux de Fondations, Montréal.

1967 Scaling laws in soil mechanics. Conférence au Congrès Panaméricain de Mécanique des Sols, Caracas.

1968 (with M. Quatre) Settlements under foundations: calculation using the triaxial apparatus. Civil Engineering, mai-juin.

1969 (with M. Adam) Charges limites d'un pieu en milieux argileux et limoneux. Comptes-Rendus du 7ème Congrès International de Mécanique des Sols et des Travaux de Fondations, Mexico.

1972 Le langage des modèles en mécanique des sols. Comptes-Rendus du 5ème Congrès Européen de Mécanique des Sols et des Travaux de Fondations, Madrid 1.

1972 Mesure de poussée et de butée faite avec 42 paires de butons asservis. Comptes-Rendus du 5ème Congrès Européen de Mécanique des Sols et des Travaux de Fondations, Madrid 1.

1973 Etat actuel de nos connaissances en mécanique des sols, lacunes et progrès. Association Belge pour 1'étude, 1'essai et 1'emploi des matériaux, publication A.B.E.M. no. 407.

1973 Le barrage d'Arzal: un barrage sur sol très compressible construit au travers d'un estuaire à marée. Géotechnique 23, No. 1, 49-65.

1973 Contraintes exercées par le sol sur la station Auber, Paris. Comptes-Rendus du 8ème Congrès International de Mécanique des sols et des Travaux de Fondations, Moscou.

1973 Bicentenaire de 1'essai de 1773 de Charles Augustin Coulomb. Introduction au 8ème Congrès International 
de Mécanique des Sols et des Travaux de Fondations, Moscou, octobre.

1975 Old structures in relation to soil conditions, $15^{\text {th }}$ Rankine Lecture. Géoechnique 25, No. 3, 433-483.

1975 Problemi del porto de Sibari, Associazione Geotecnica Italiana, XII convegno nazionale di geotecnica, Cosenza, settembre.

1976 Transmission des surcharges à travers un massif, Annales de 1'Institut Technique du Bâtiment et des Travaux Publics, supplement au no. 335.

1977 (with P. Savey) Soulèvements d'une argile dechargée et leur prevision. Comptes-Rendus 9ème Congrès International de Mécanique des Sols et des Travaux de Fondations, Tokyo.

1978 Exploitation de 1'énergie marémotrice disponible dans la baie du Mont Saint Michel. Exposé devant 1'Académie des Sciences, Paris.

1978 (with R. Persoz) Coulomb et la rhéologie. Industrie Minérale, 15 juin (also published in 1979 by the British Society of Rheology).

1978 Méthode convergence-confinement pour le calcul des revêtements de tunnels.

1981 Quelques reflexions sur l'interaction sol-structure. Collège International des Sciences du Bâtiment.

1982 Les chantiers et monuments du passé: leçons pour 1'ingénieur et 1'architecte; Panama, Pantheon, Pise, Chine du 11ème siecle. Annales de I'Institut Technique du Bâtiment et des Travaux Publics 410, 3780.

1982 Perspectiva historica de la ingenieria geotecnica. Real Academia de Ciencias Exactas, Fisicas y Naturales, Madrid.

1983 Les restaurations à travers le temps, un facteur important: le sol. Exemple d'une restauration célèbre: celle du Panthéon français. Conférence à l'Ecole Polytechnique, Paris.
1985 Histoire de 1'ingénierie geomécanique jusqu'à 1700 . Livre d'Or sur l'Histoire de la Geomécanique, 50ème Anniversaire de la SIMSTF, 11ème Congrès International de Mécanique des Sols et des Travaux de Fondations, San Francisco.

1985 Evaluation of the small cohesion existing in natural sands deemed to be cohesionless. Acta Tecnica Academiae Scientiarum Hungaricae 98, No. 1-2, 87-95.

1988 Civilisation et monuments. Exposé devant 1'Académie des Sciences Morales et Politiques, Paris.

1988 A glimpse of geotechnical engineering during the fourth and third millennia BC. Comptes-Rendus d'un Symposium International organisé par le groupe national grec de l'I.A.E.G. Balkema: Rotterdam.

1992 History of retaining walls design. Proceedings of the conference on retaining structures, Cambridge, pp. 1-16. London: Thomas Telford.

2000 History of retaining wall design. Geotecnica en el año 2000. Libro homenaje à Jose Antonio Jimenez Salas.

Papers in Egyptology

1984 L'invention dans la pyramide egyptiénne. Imhotep et ses successeurs. Symposium Interdisciplinaire de Sciences Humaines et Physiques, avril, Université de Naples.

1996 Liaisons interocéaniques des XIXè et XXè siècles: Suez et Panama, lointaines conséquences de l'expédition d'Egypte. De Jomard (1794) à Bunau-Varilla (1878) en passant par Godin de Lépinay (1840). Revue la Jaune et la Rouge, no. 512, 53-59.

2002 The tomb of Cheops and the testimony of Herodotus. Discussions in Egyptology 53, 47-55.

2003 Le tombeau de Khéops et une vérification peu coûteuse. Revue Travaux, no. 796, 68-76.

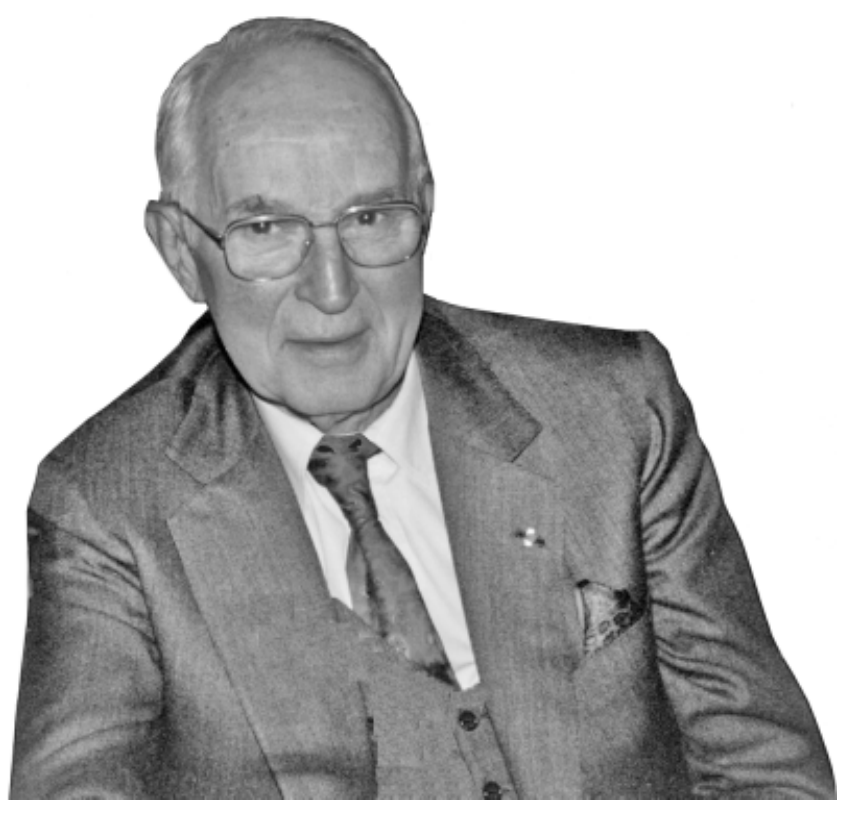

Jean Kérisel, 1908-2005 